\title{
ON THE WEAK BEHAVIOUR OF PARTIAL SUMS OF LEGENDRE SERIES
}

BY

S. CHANILLO

ABSTRACT. We show that the partial sum operator associated with the Legendre series is restricted weak type, but not weak type, on the $L^{p}$ spaces when $p=4$.

Introduction. Let $P_{n}(x)$ denote the $n$th Legendre function. A function $f(x)$ such that $f \in L^{1}(-1,1)$ then can be expanded formally in a Legendre series, i.e.

$$
f(x) \sim \sum_{n=0}^{\infty} a_{n} P_{n}(x), \quad \text { where } a_{n}=\left(n+\frac{1}{2}\right) \int_{-1}^{1} f(t) P_{n}(t) d t .
$$

$S_{N}(f)(x)$ denotes the $N$ th partial sum of the Legendre expansion of $f(x)$. It was proved by $H$. Pollard in [4] that if $f \in L^{p}(-1,1)$, where $4 / 3<p<4$, then one gets mean convergence in $L^{p}$, i.e. $\left\|S_{N}(f)\right\|_{p}<C_{p}\|f\|_{p}$, where $C_{p}$ is independent of $N$ and $f(x)$. He also showed that mean convergence fails if $p<4 / 3$ or $p>4$. However J. Newman and W. Rudin in [3] showed that the inequality $\left\|S_{N}(f)\right\|_{p}<$ $C_{p}\|f\|_{p}$ is false at $p=4$ or $p=4 / 3$. The aim of this paper is to examine the weak behaviour at $p=4$, much in the spirit of the Hilbert transform at $L^{1}(0,2 \pi)$.

We now need some notations to state our main results. The Lebesgue measure of any set $E \subseteq(-1,1)$ will be denoted as $m(E) . \chi_{E}(x)$ will as usual denote the characteristic function of a set $E$. We then have

THEOREM 1. Let $S_{N}\left(\chi_{E}\right)(x)$ denote the Nth partial sum of the Legendre series of $\chi_{E}(x)$; then

$$
m\left\{x:\left|S_{N}\left(\chi_{E}\right)(x)\right|>y\right\} \leqslant\left(C / y^{4}\right)(m(E)), \quad x \in(-1,1), \quad y>0 .
$$

The constant $C$ is independent of $N$ and the set $E$.

THEOREM 2. Let $f \in L^{4}(-1,1)$; then there exists no constant $C$, independent of $N$ and $f$, such that

$$
m\left\{x:\left|S_{N}(f)(x)\right|>y\right\} \leqslant\left(C / y^{4}\right)\|f\|_{4}^{4}, \quad x \in(-1,1),
$$

and

$$
\|f\|_{4}^{4}=\left(\int_{-1}^{1}|f|^{4} d t\right)
$$

In other words the partial sum operator is restricted weak type $(4,4)$, but not weak type $(4,4)$.

Received by the editors October 20, 1980.

1980 Mathematics Subject Classification. Primary 42A56, 44A25, 42A40; Secondary 43A90. 
The proof of Theorem 1 uses some elements of the theory of $A_{p}$ weights. In fact the result of $\mathrm{H}$. Pollard could have been deduced from the results of Hunt, Muckenhoupt and Wheeden in [2] on the Hilbert transform.

We wish to thank Professor H. Pollard for his encouragement while working on this problem.

Proof of Theorem 1. We shall begin with a partial sum formula for $S_{N}(f)(x)$, which is obtained by a rearrangement of the Christoffel identity and was obtained by Pollard [4].

$$
\begin{aligned}
S_{N}(f)(x)= & \left(\frac{N+2}{2 N+3}\right)\left(\frac{N+1}{2}\right) \cdot P_{N+1}(x) \cdot \int_{-1}^{1} \frac{P_{N}(t)-P_{N+2}(t)}{x-t} f(t) d t \\
& +\left(\frac{N+2}{2 N+3}\right)\left(\frac{N+1}{2}\right)\left(P_{N+2}(x)-P_{N}(x)\right) \int_{-1}^{1} \frac{P_{N+1}(t) f(t) d t}{x-t} \\
& -\left(\frac{N+1}{2}\right) \cdot P_{N+1}(x) \cdot \int_{-1}^{1} P_{N+1}(t) f(t) d t .
\end{aligned}
$$

The symbol $C$ will denote a constant different in different places but independent of $N$ and the set $E$.

We shall also need some estimates on the Legendre polynomials, which are readily available in the book of $\mathrm{G}$. Szegö [6].

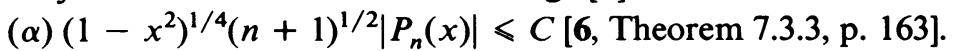

( $\beta)\left(1-x^{2}\right)^{-1 / 4}(n+1)^{1 / 2}\left|P_{n}(x)-P_{n+2}(x)\right| \leqslant C[6,7.33 .10$, p. 170].

We now try to estimate the terms of equation (1).

We shall get rid of the middle term by using the weight results on the Hilbert transform and the last term is estimated by Hölder's inequality.

We begin by estimating the middle term by using $(\beta)$.

$$
\begin{aligned}
& \left|\left(\frac{N+2}{N+3}\right) \frac{N+1}{2}\left(P_{N+2}(x)-P_{N}(x)\right) \int_{-1}^{1} \frac{P_{N+2}(t) f(t) d t}{x-t}\right| \\
& \leqslant C(N+1)^{1 / 2}\left(1-x^{2}\right)^{1 / 4}\left|H\left(P_{N+2}(t) f(t)\right)(x)\right|
\end{aligned}
$$

where $H$ is the Hilbert transform.

Now by the Chebyshev inequality from (2) we get

$$
\begin{aligned}
m\left\{x:(N+1)^{1 / 2}\left(1-x^{2}\right)^{1 / 4}\left|H\left(P_{N+2}(t) f(t)\right)(x)\right|>y\right\} \\
\leqslant \frac{1}{y^{4}} \int_{-1}^{1}(N+1)^{2}\left|H\left(P_{N+2}(t) f(t)\right)(x)\right|^{4}\left(1-x^{2}\right) d x
\end{aligned}
$$

We now note that the function $\left(1-x^{2}\right)$ is readily seen to belong to the weight class $\boldsymbol{A}_{4}$ (see [2]). Consequently the theorem of Hunt, Muckenhoupt and Wheeden [2] yields that the right-hand side of (3) is majorized by

$$
\frac{(N+1)^{2}}{y^{4}} \int_{-1}^{1}|f(x)|^{4}\left|P_{N+2}(x)\right|^{4}\left(1-x^{2}\right) d x .
$$


Using the estimate $(\alpha)$, the integral of (4) is majorized by

$$
\frac{C}{y^{4}} \int_{-1}^{1}|f(x)|^{4} d x=C \frac{\|f\|_{4}^{4}}{y^{4}}
$$

the desired estimate.

We now estimate the last term in (1). Now

$$
\begin{aligned}
& \frac{(N+1)}{2}\left|P_{N+1}(x)\right| \mid \\
& \int_{-1}^{1} P_{N+1}(t) f(t) d t \mid \\
& \leqslant C(N+1)^{1 / 2}\left(1-x^{2}\right)^{-1 / 4} \int N^{-1 / 2}|f(t)|\left(1-t^{2}\right)^{-1 / 4} d t .
\end{aligned}
$$

The last expression follows by use of the estimate $(\alpha)$. Using Hölder's inequality on the right-hand side of (5) we estimate (5) by $C\left(1-x^{2}\right)^{-1 / 4}\|f\|_{4}$. Now

$$
m\left\{x: C\left(1-x^{2}\right)^{-1 / 4}\|f\|_{4}>y\right\}<\left(C / y^{4}\right)\|f\|_{4}^{4}
$$

which is the desired estimate.

So now we only have to estimate the first term in (1). It is only here that the assumption $f(x)=\chi_{E}(x)$ becomes important. All of what we have done so far works for any general function $f \in L^{4}(-1,1)$.

Using the estimate ( $\alpha)$, the first term in (1) is majorized by

$$
C(N+1)^{1 / 2}\left(1-x^{2}\right)^{-1 / 4}\left|H\left(\chi_{E}(t)\left(P_{N}(t)-P_{N+2}(t)\right)\right)(x)\right| .
$$

We will show that

$m\left\{x:(N+1)^{1 / 2}\left(1-x^{2}\right)^{-1 / 4}\left|H\left(\chi_{E}(t)\left(P_{N}(t)-P_{N+2}(t)\right)\right)(x)\right|>y\right\}$

The estimate (6), then, along with the estimates for the middle and last expressions in (1), which we have already proved, then yields Theorem 1.

Before we make any decompositions of sets and functions in the left-hand side of (6), we will make some reductions. We will denote by $U$ the set occurring in the left-hand side of (6). We also decompose $E=E_{1} \cup E_{2}, E_{1}=E \cap[0,1)$, and $E_{2}=(-1,0) \cap E$.

Now we note that

$m\left(U \cap\left\{x:|x| \leqslant \frac{3}{4}\right\}\right) \leqslant m\left\{x: C(N+1)^{1 / 2}\left|H\left(\chi_{E}(t)\left(P_{N}(t)-P_{N+2}(t)\right)\right)(x)\right|>y\right\}$.

Using the classical result of $M$. Riesz [7, p. 253] we get that the right-hand side of the last inequality is majorized by

$$
C \frac{(N+1)^{2}}{y^{4}} \int\left|\chi_{E}\right|^{4}\left|P_{N}(t)-P_{N+2}(t)\right|^{4} d t .
$$

The estimate $(\beta)$ then is used to majorize (7) by $C \cdot m(E) / y^{4}$. Consequently Theorem 1 will be proved if we show that

$m\left\{x: \frac{3}{4}<|x|<1,(N+1)^{1 / 2}\left(1-x^{2}\right)^{-1 / 4}\left|H\left(\left(P_{N}(t)-P_{N+2}(t)\right) \chi_{E_{1}}(t)\right)(x)\right|>y\right\}$ 
and

$m\left\{x: \frac{3}{4}<|x|<1,(N+1)^{1 / 2}\left(1-x^{2}\right)^{-1 / 4}\left|H\left(\left(P_{N}(t)-P_{N+2}(t)\right) \chi_{E_{2}}(t)\right)(x)\right|>y\right\}$

$$
\leqslant C m(E) / y^{4} \text {. }
$$

We will only prove (8). The estimates for proving (9) are made in the same manner as (8). We can now make a further reduction in proving (8). We next show that

$m\left\{x:-1<x<-\frac{3}{4},(N+1)^{1 / 2}\left(1-x^{2}\right)^{-1 / 4}\left|H\left(\left(P_{N}(t)-P_{N+2}(t)\right) \chi_{E_{1}}(t)\right)(x)\right|>y\right\}$

$$
\leqslant C m(E) / y^{4} \text {. }
$$

This is because if $x \in(-1,-3 / 4)$ and $E_{1} \subseteq[0,1)$,

$$
\begin{aligned}
\left|H\left(\left(P_{N}(t)-P_{N+2}(t)\right) \chi_{E_{1}}(t)\right)(x)\right| & =\left|\int_{-1}^{1} \frac{\chi_{E_{1}}(t)\left(P_{N}(t)-P_{N+2}(t)\right)}{x-t} d t\right| \\
& \leqslant C \int_{-1}^{1}\left|\chi_{E_{1}}(t)\right|\left|P_{N}(t)-P_{N+2}(t)\right| d t .
\end{aligned}
$$

Using the estimate $(\beta)$ we majorize by

$$
C(N+1)^{-1 / 2} \int_{-1}^{1}\left|\chi_{E_{1}}(t)\right| d t \leqslant C(N+1)^{-1 / 2} m(E)^{1 / 4}
$$

Hence the left-hand side of $(10)$ is majorized by

$$
m\left\{x:\left(1-x^{2}\right)^{-1 / 4}(m(E))^{1 / 4}>y\right\} .
$$

This is estimated by $C m(E) / y^{4}$. So it is enough to prove that

$m\left\{x: \frac{3}{4}<x<1,(N+1)^{1 / 2}\left(1-x^{2}\right)^{-1 / 4}\left|H\left(\left(P_{N}(t)-P_{N+2}(t)\right) \chi_{E_{1}}(t)\right)(x)\right|>y\right\}$

$$
\leqslant C m(E) / y^{4} \text {. }
$$

Let us denote by $A$ the set appearing on the left-hand side of (11). Let

$$
\begin{aligned}
& I_{k}^{0}=\left\{x: 0 \leqslant x \leqslant 1-2^{-k+1}, k \geqslant 2\right\} \\
& I_{k}=\left\{x: 2^{-k-1}<1-x \leqslant 2^{-k}, k \geqslant 2\right\} \\
& \tilde{I}_{k}=\left\{x: 2^{-k-2}<1-x<2^{-k+1}, k \geqslant 2\right\} \\
& \hat{I}_{k}=\left\{x: 1-2^{-k-2} \leqslant x<1, k \geqslant 2\right\}
\end{aligned}
$$

Let $A_{k}=A \cap I_{k}$. Then $A=\cup_{k=2}^{\infty} A_{k}$, and $A_{k} \cap A_{j}=\varnothing$ if $k \neq j$. We, moreover, decompose $\chi_{E_{1}}(t)$ as follows. has

Let $\chi_{E_{11}}^{(k)}(t)=\chi_{E_{1}}(t) \chi_{I_{k}^{0}}(t), \chi_{E_{12}}^{(k)}(t)=\chi_{E_{1}}(t) \chi_{I_{k}}(t), \chi_{E_{13}}^{(k)}(t)=\chi_{E_{1}}(t) \chi_{\hat{I}_{k}}(t)$. Clearly one

$$
\chi_{E_{1}}(t)=\chi_{E_{12}}^{(k)}(t)+\chi_{E_{12}}^{(k)}(t)+\chi_{E_{13}}^{(k)}(t)
$$


Let us also set for $k \geqslant 2$ :

$$
\begin{aligned}
& A_{k}^{(1)}=\left\{x: 2^{-k-1}<1-x \leqslant 2^{-k},(N+1)^{1 / 2}\left(1-x^{2}\right)^{-1 / 4}\right. \\
& \left.\cdot\left|H\left(\chi_{E_{11}}^{(k)}(t)\left(P_{N}(t)-P_{N+2}(t)\right)\right)(x)\right|>y / 3\right\} ; \\
& A_{k}^{(2)}=\left\{x: 2^{-k-1}<1-x \leqslant 2^{-k},(N+1)^{1 / 2}\left(1-x^{2}\right)^{-1 / 4}\right. \\
& \left.\cdot\left|H\left(\chi \sum_{12}^{(k)}(t)\left(P_{N}(t)-P_{N+2}(t)\right)\right)(x)\right|>y / 3\right\} ; \\
& A_{k}^{(3)}=\left\{x: 2^{-k-1}<1-x \leqslant 2^{-k},(N+1)^{1 / 2}\left(1-x^{2}\right)^{-1 / 4}\right. \\
& \left.\cdot\left|H\left(\chi_{E_{13}}^{(k)}(t)\left(P_{N}(t)-P_{N+2}(t)\right)\right)(x)\right|>y / 3\right\} .
\end{aligned}
$$

We shall get rid of $A_{k}^{(1)}$ and $A_{k}^{(3)}$ first, the estimates involved are also similar. Also we note that $A_{k} \subseteq A_{k}^{(1)} \cup A_{k}^{(2)} \cup A_{k}^{(3)}$. We will show that

$$
A_{k}^{(1)} \cup A_{k}^{(2)} \subseteq\left\{x: C(m(E))^{1 / 4}\left(1-x^{2}\right)^{-1 / 4}>y\right\} .
$$

To do so it is enough to show that if $x \in I_{k}$ then

$$
\begin{aligned}
&(N+1)^{1 / 2}\left(1-x^{2}\right)^{-1 / 4}\left|H\left(\chi_{E_{11}}^{(k)}(t)\left(P_{N}(t)-P_{N+2}(t)\right)\right)(x)\right| \\
& \leqslant C(m(E))^{1 / 4}\left(1-x^{2}\right)^{-1 / 4}
\end{aligned}
$$

and

$$
\begin{gathered}
(N+1)^{1 / 2}\left(1-x^{2}\right)^{-1 / 4}\left|H\left(\chi_{E_{13}}^{(k)}(t)\left(P_{N}(t)-P_{N+2}(t)\right)\right)(x)\right| \\
\leqslant C(m(E))^{1 / 4}\left(1-x^{2}\right)^{-1 / 4}
\end{gathered}
$$

By the location of the support of $\chi_{E_{11}}^{(k)}(t)$ and the geometry of the situation, the left-hand side of (12) is majorized by

$$
\begin{aligned}
& (N+1)^{1 / 2}\left(1-x^{2}\right)^{-1 / 4} \int_{-1}^{1} \frac{\chi_{E_{11}}^{(k)}(t)\left|P_{N}(t)-P_{N+2}(t)\right| d t}{|x-t|} \\
& \leqslant C(N+1)^{1 / 2}\left(1-x^{2}\right)^{-1 / 4} \int_{-1}^{1} \frac{\chi_{E_{11}}^{(k)}(t)\left|P_{N}(t)-P_{N+2}(t)\right| d t}{|1-t|}
\end{aligned}
$$

Using the estimate $(\beta)$ we get

$$
\leqslant C\left(1-x^{2}\right)^{-1 / 4} \int_{-1}^{1} \chi_{E_{11}}^{(k)}(t)(1-t)^{-3 / 4} d t
$$

We now use Hölder's inequality for Lorentz spaces. This is Theorem 4.5 in Hunt [1]. The integral of (14) is then estimated by

$$
\begin{aligned}
& \leqslant C \cdot\left(1-x^{2}\right)^{-1 / 4}\left\|\chi_{E(\xi)}\right\|_{(4,1)}\left\|(1-t)^{-3 / 4}\right\|_{(4 / 3, \infty)} \\
& \leqslant C\left(1-x^{2}\right)^{-1 / 4}(m(E))^{1 / 4} .
\end{aligned}
$$


Similarly if $x \in I_{k}$, by the geometry,

$$
\begin{aligned}
\left|H\left(\chi \chi_{E_{13}}^{(k)}(t)\left(P_{N}(t)-P_{N+2}(t)\right)\right)(x)\right| & \leqslant \int_{-1}^{1} \frac{\chi_{E_{13}}^{(k)}(t)\left|P_{N}(t)-P_{N+2}(t)\right| d t}{|x-t|} \\
& \leqslant C \int_{-1}^{1} \chi_{E_{13}}^{(k)}(t) \frac{\left|P_{N}(t)-P_{N+2}(t)\right| d t}{|1-t|} .
\end{aligned}
$$

From the estimate $(\beta)$ we get

$$
\leqslant C(N+1)^{-1 / 2} \int_{-1}^{1} \chi_{E_{13}}^{(k)}(t)(1-t)^{-3 / 4} d t .
$$

Again invoking Hölder's inequality for Lorentz spaces we majorize (15) by $C(N+1)^{-1 / 2}(m(E))^{1 / 4}$. Consequently we have shown that

$$
A_{k}^{(1)} \cup A_{k}^{(3)} \subseteq\left\{x: C\left(1-x^{2}\right)^{-1 / 4}(m(E))^{1 / 4}>y\right\} .
$$

Now

$$
m(A) \leqslant m\left(\bigcup_{k=2}^{\infty}\left(A_{k}^{(1)} \cup A_{k}^{(3)}\right)\right)+\sum_{k=2}^{\infty} m\left(A_{k}^{(2)}\right)
$$

Now we note that

$$
A_{k}^{(2)} \subseteq\left\{x:(N+1)^{1 / 2}\left|H\left(\chi_{E_{12}}^{(k)}(t)\left(P_{N}(t)-P_{N+2}(t)\right)\right)(x)\right|>C y \cdot 2^{-k / 4}\right\} .
$$

The result of $M$. Riesz once again yields that

$$
m\left(A_{k}^{(2)}\right) \leqslant \frac{C}{y^{4}} \cdot 2^{k} N^{2} \int \chi_{E_{12}}^{(k)}(t)\left|P_{N}(t)-P_{N}(t)\right|^{4} d t
$$

By the location of the support of $\chi_{E_{12}}^{(k)}(t)$ and the estimate $(\beta)$ we have (18) majorized by

$$
\frac{C}{y^{4}} \int \chi_{E_{12}}^{(k)}(t) d t
$$

Substituting (16) and the estimate (19) for $m\left(A_{k}^{(2)}\right)$ into (17) we get

$$
m(A) \leqslant m\left\{x: C(m(E))^{1 / 4}\left(1-x^{2}\right)^{-1 / 4}>y\right\}+\sum_{k=2}^{\infty} \frac{C}{y^{4}} \int \chi_{E_{12}}^{(k)}(t) d t .
$$

By the fact that the supports of $\chi_{E_{13}}^{(k)}(t)$ have bounded overlap (in fact a point may belong to at most eight intervals $\left.\tilde{I}_{k}\right)$, we get $m(A) \leqslant C m(E) / y^{4}$. Q.E.D.

Before proving Theorem 2 we wish to make some remarks. The first one is that while proving Theorem 1 , the only place we really needed characteristic functions was when we used Hölder's inequality for Lorentz spaces. It is this fact along with sharper estimates instead of $(\alpha)$ and $(\beta)$ which, when exploited, give us Theorem 2. Of course one would like to show Theorem 2 by trying to mimic the elegant functional analysis method of J. Newman and W. Rudin [3]. This, however, breaks down. Keeping this in mind the idea behind Theorem 2 is almost clear. 
Proof of Theorem 2. The proof of this theorem is by contradiction. So let us assume that there exists a constant $C$, independent of $N$, and $f \in L^{4}(-1,1)$ such that

$$
m\left\{x:\left|S_{N}(f)(x)\right|>y\right\} \leqslant\left(C / y^{4}\right)\|f\|_{4}^{4}, \quad y>0, \quad x \in(-1,1) .
$$

By (1) and the first part of Theorem 1 it follows that the above statement implies

$$
m\left\{x: N\left|P_{N}(x)\right|\left|H\left(f(t)\left(P_{N}(t)-P_{N+2}(t)\right)\right)(x)\right|>y\right\} \leqslant C\|f\|_{4}^{4} / y^{4} .
$$

So it will be enough to construct a sequence of functions $\left\{f_{N}(t)\right\}$ such that the constant $C$ appearing in the inequality above grows with $N$.

We now collect the asymptotic formulae we need from the book of G. Szegö [6]. As indicated above these are sharper forms of $(\alpha)$ and $(\beta)$. The first one is obtained from Theorem 8.21.13, p. 195 of [6]. Hence we have

$$
P_{n}(\operatorname{Cos} \theta)=n^{-1 / 2} k(\theta)\left[\operatorname{Cos}\left(\left(n+\frac{1}{2}\right) \theta-\frac{\pi}{4}\right)+(n \operatorname{Sin} \theta)^{-1} O(1)\right]
$$

where

$$
k(\theta)=(\pi / 2)^{-1 / 2}(\operatorname{Sin} \theta)^{-1 / 2} \text { and } c n^{-1} \leqslant \theta \leqslant \pi-c n^{-1},
$$

$c$ being a fixed positive number.

In our application though we will restrict our attention to $\theta \leqslant \pi / 2$. We now develop the estimates for $P_{n}(x)-P_{n+2}(x)$. By equation 7.33.9, p. 170 of [6],

$$
\left(1-x^{2}\right) P_{n+1}^{\prime}(x)=(n(n+1) / 2 n+1)\left(P_{n}(x)-P_{n+2}(x)\right) \text {. }
$$

Hence, by equation 4.21 .7 , p. 63 of [5],

$$
\left(1-x^{2}\right) P_{n+1}^{\prime}(x)=\left(1-x^{2}\right)((n+2) / 2) P_{n}^{(1,1)}(x) \text {. }
$$

So we get that

$$
\left|P_{n}(x)-P_{n+2}(x)\right| \geqslant b\left(1-x^{2}\right)\left|P_{n}^{(1,1)}(x)\right| .
$$

As remarked previously we only need the part when $\theta \leqslant \pi / 2$. We now employ the "Hilb's type" result, Theorem 8.21.12, p. 195 of [6]. Substituting $\alpha=\beta=1$ in the formula we get

$$
\begin{aligned}
(\operatorname{Sin} \theta) P_{n}^{(1,1)}(\operatorname{Cos} \theta)= & 2\left(n+\frac{3}{2}\right)^{-1}(n+1)\left(\frac{\theta}{\operatorname{Sin} \theta}\right)^{1 / 2} J_{1}\left(\left(n+\frac{3}{2}\right) \theta\right) \\
& +\theta^{1 / 2} O\left(n^{-3 / 2}\right)
\end{aligned}
$$

where $c n^{-1} \leqslant \theta \leqslant \pi / 2, c$ is a fixed positive number. $J_{1}$ is the Bessel function of order one. We shall now make some observations from (21). Let us choose $M$ large enough and integral. The choice of $M$ is determined by what follows. $M$ is then fixed for all choices of $n$. Consider then $\theta$ such that $M \pi / n \leqslant \theta \leqslant\left(M+\frac{1}{8}\right) \pi / n$. In this range of $\theta,(21)$ yields

$$
\left|P_{n}(\operatorname{Cos} \theta)\right| \geqslant C \cdot n^{-1 / 2}|\operatorname{Sin} \theta|^{-1 / 2}|| \operatorname{Cos}\left(M \pi+\frac{M \pi}{2 n}-\frac{\pi}{8}\right)\left|-\frac{c}{M \pi}\right|, \quad n \geqslant N_{0} .
$$

If $M$ is taken sufficiently large we get

$$
\left|P_{n}(\operatorname{Cos} \theta)\right| \geqslant C n^{-1 / 2}|\operatorname{Sin} \theta|^{-1 / 2}, \quad n \geqslant N_{0}, \quad \theta \in\left(M \pi / n,\left(M+\frac{1}{8}\right) \pi / n\right) .
$$


If one writes $x=\operatorname{Cos} \theta$ we get

$$
\begin{aligned}
& \left|P_{n}(x)\right| \geqslant C n^{-1 / 2}\left(1-x^{2}\right)^{-1 / 4} \\
& \qquad n \geqslant N_{0}, \quad x \in\left(\operatorname{Cos} \frac{(M+1 / 8) \pi}{n}, \operatorname{Cos} \frac{(M \pi)}{n}\right) .
\end{aligned}
$$

We define

$$
f_{N}(t)= \begin{cases}\frac{\operatorname{sgn}\left(P_{N}(t)-P_{N+2}(t)\right)}{\left(1-t^{2}\right)^{1 / 4}}, & 0 \leqslant t \leqslant \operatorname{Cos}\left(\frac{2 M \pi}{N}\right), \\ 0, & \text { elsewhere. }\end{cases}
$$

Note now $N \geqslant N_{0}$. We will now estimate the Hilbert transform expression in (20).

$$
\left|H\left(f_{N}(t)\left(P_{N}(t)-P_{N+2}(t)\right)\right)(x)\right|, \quad x \in\left(\operatorname{Cos}\left(\frac{(M+1 / 8) \pi}{N}\right), \operatorname{Cos}\left(\frac{M \pi}{N}\right)\right)=I_{N},
$$

where $H$ is the Hilbert transform

$$
=\left|\int_{0}^{\operatorname{Cos}(2 M \pi / N)} \frac{\left|P_{N}(t)-P_{N+2}(t)\right|}{\left(1-t^{2}\right)^{1 / 4}(x-t)} d t\right| .
$$

If now $x \in I_{N}$ and $0 \leqslant t \leqslant \operatorname{Cos}(2 M \pi / N)$, then $t \leqslant x$ and $(x-t) \leqslant C(1-t)$; the last inequality is an easy consequence of the half-angle formulae. Hence (25) is bigger than

$$
C \int_{0}^{\operatorname{Cos}(2 M \pi / N)} \frac{\left|P_{N}(t)-P_{N+2}(t)\right| d t}{\left(1-t^{2}\right)^{1 / 4}\left(1-t^{2}\right)} .
$$

Making the change of variable $t=\operatorname{Cos} \theta$ in the last integral we get

$$
C \int_{2 M \pi / N}^{\pi / 2} \frac{\left|P_{N}(\operatorname{Cos} \theta)-P_{N+2}(\operatorname{Cos} \theta)\right| d \theta}{(\operatorname{Sin} \theta)^{3 / 2}} .
$$

Now from (22) and (23) it follows that (26) is bounded below by

$$
\begin{aligned}
& \geqslant \int_{2 M \pi / N}^{\pi / 2} \frac{\left|J_{1}((N+3 / 2) \theta)\right|}{(\operatorname{Sin} \theta)^{1 / 2}} d \theta-C \int_{2 M \pi / N}^{\pi / 2} \frac{\theta^{1 / 2} N^{-3 / 2}}{(\operatorname{Sin} \theta)^{1 / 2}} d \theta \\
& \geqslant C \int_{2 M \pi / N}^{\pi / 2} \frac{\left|J_{1}((N+3 / 2) \theta)\right|}{\theta^{1 / 2}} d \theta-c N^{-3 / 2}
\end{aligned}
$$

One can now use the asymptotics of the Bessel function at infinity since $(N+3 / 2) \theta \geqslant c M \pi, M$ being large. The asymptotics are found in [6, equation 1.71.7, p. 15].

$$
J_{1}(x)=-(2 / \pi x)^{1 / 2} \operatorname{Sin}(x-\pi / 4)+O\left(x^{-3 / 2}\right), \quad x \rightarrow+\infty .
$$

Substituting these asymptotics into (27) we get

$$
\begin{gathered}
C N^{-1 / 2} \int_{2 M \pi / N}^{\pi / 2} \frac{|\operatorname{Sin}((N+3 / 2) \theta-\pi / 4)|}{\theta} d \theta-c N^{-3 / 2} \int_{2 M \pi / N}^{\pi / 2} \frac{d \theta}{\theta^{2}}-c N^{-3 / 2} \\
\quad=c_{1} N^{-1 / 2} \log N-c_{2} N^{-1 / 2}-c_{3} N^{-3 / 2} \\
\geqslant c N^{-1 / 2} \log N \quad \text { for } N \geqslant N_{0} .
\end{gathered}
$$


So finally we have shown that if $x \in I_{N}$ and $f_{N}(t)$ is as defined, then

$$
\left|H\left(f_{N}(t)\left(P_{N}(t)-P_{N+2}(t)\right)\right)(x)\right| \geqslant C N^{-1 / 2} \log N .
$$

We also find that

$$
\int_{-1}^{1}\left|f_{N}(t)\right|^{4} d t \leqslant \int_{0}^{\operatorname{Cos}(2 M \pi / N)} \frac{d t}{\left(1-t^{2}\right)}
$$

Making the change of variable $t=\operatorname{Cos} \theta$ we get

$$
\leqslant \int_{2 M \pi / N}^{1} \frac{d \theta}{\operatorname{Sin} \theta} \leqslant c \int_{2 M \pi / N}^{1} \frac{d \theta}{\theta} \leqslant c(\log N) .
$$

From (20) it follows that

$$
\begin{gathered}
m\left\{x: x \in I_{N}, N\left|P_{N}(x)\right|\left|H\left(f_{N}(t)\left(P_{N}(t)-P_{N+2}(t)\right)\right)(x)\right|>y\right\} \\
\leqslant\left(C / y^{4}\right)\left\|f_{N}\right\|_{4}^{4} .
\end{gathered}
$$

Now by (24) it follows that if $x \in I_{N}$, then

$$
\begin{aligned}
\left|P_{N}(x)\right| & \geqslant C N^{-1 / 2}\left(1-\operatorname{Cos}^{2}(M \pi / N)\right)^{-1 / 4} \\
& \geqslant C N^{-1 / 2} \operatorname{Sin}^{-1 / 2}(M \pi / N)>C, \quad N \geqslant N_{0} .
\end{aligned}
$$

Hence by (28) it then follows that if $x \in I_{N}$,

$$
N\left|P_{N}(x)\right|\left|H\left(f_{N}(t)\left(P_{N}(t)-P_{N+2}(t)\right)\right)(x)\right| \geqslant C N^{1 / 2}(\log N) .
$$

Choose $y=c N^{1 / 2}(\log N)$. Note $m\left(I_{N}\right) \geqslant C / N^{2}, N \geqslant N_{0}$. We then have from (30), using estimate (29),

$$
\frac{C}{N^{2}} \leqslant \frac{C}{N^{2}(\log N)^{4}} \log N, \quad \text { or } \quad c_{1} \leqslant c_{2}(\log N)^{-3}, \quad N \geqslant N_{0} .
$$

This is a contradiction. Q.E.D.

It is very likely that most of our results would carry over to the ultraspherical case. But maybe by perturbing the weight functions one could get weak type results in that context. The meaning of our result in the context of spherical functions is also at the moment unclear.

We would also like to point out an answer to a question raised by $\mathrm{H}$. Pollard, which is to consider the maximal function $x \in(-1+\varepsilon, 1-\varepsilon), \sup _{N}\left|S_{N} f(x)\right|=$ $f^{*}(x)$, then to show if $\left\|f^{*}\right\|_{p} \leqslant C(\varepsilon)\|f\|_{p}, 4 / 3<p<4$. The answer to this question is in the affirmative. It follows by using the Haar equiconvergence theorem [6, Theorem 9.1.2, p. 244] and weighted norm inequalities for the maximal partial sum operator for Fourier series due to R. A. Hunt and Wo-Sang Young. The norm estimate above easily yields the pointwise convergence of Legendre series. This fact in itself had been established by $\mathrm{H}$. Pollard in [5].

ADDED IN PROOF. Theorem 1 is valid when $p=4 / 3$. 


\section{REFERENCES}

1. R. A. Hunt, On $L(p, q)$ spaces, Enseign. Math. 12 (1966), 249-275.

2. R. A. Hunt, B. Muckenhoupt and R. L. Wheeden, Weighted norm inequalities for the conjugate function and the Hilbert transform, Trans. Amer. Math. Soc. 176 (1973), 227-251.

3. J. Newman and W. Rudin, Mean convergence of orthogonal series, Proc. Amer. Math. Soc. 3 (1952), 219-222.

4. H. Pollard, The mean convergence of orthogonal series. I, Trans. Amer. Math. Soc. 62 (1947), $387-403$.

5. __ The convergence almost everywhere of Legendre series, Proc. Amer. Math. Soc. 35 (1972), 442-444.

6. G. Szegö, Orthogonal polynomials, Amer. Math. Soc. Colloq. Publ., vol. 23, Amer. Math. Soc., Providence, R. I., 1959.

7. A. Zygmund, Trigonometric series. Vol. II, Cambridge Univ. Press, New York, 1977.

Department of Mathematics, Rutgers University, New Brunswick, New Jersey 08903 